LTH 692

\title{
Renormalization group aspects of the local composite operator method $\ddagger$
}

\author{
R E Browne ${ }^{1}$, D Dudal ${ }^{2}$, J A Gracey ${ }^{1}$, V E R Lemes ${ }^{3}$, M S \\ Sarandy $^{4}$, R F Sobreiro ${ }^{3}$, S P Sorella ${ }^{3}$ and H Verschelde ${ }^{2}$ \\ 1 Theoretical Physics Division, Department of Mathematical Sciences, University of \\ Liverpool, P.O. Box 147, Liverpool, L69 3BX, United Kingdom \\ ${ }^{2}$ Ghent University, Department of Mathematical Physics and Astronomy, Krijgslaan \\ 281-S9, B-9000 Gent, Belgium \\ ${ }^{3}$ Departamento de Física Teórica, Instituto de Física, UERJ, Universidade do \\ Estado do Rio de Janeiro, Rua São Francisco Xavier 524, 20550-013 Maracanã, Rio \\ de Janeiro, Brazil \\ ${ }^{4}$ Chemical Physics Theory Group, Department of Chemistry, University of Toronto, \\ 80 St George Street, Toronto, Ontario, M5S 3H6, Canada \\ E-mail: jag@amtp.liv.ac.uk
}

\begin{abstract}
We review the current status of the application of the local composite operator technique to the condensation of dimension two operators in quantum chromodynamics (QCD). We pay particular attention to the renormalization group aspects of the formalism and the renormalization of $\mathrm{QCD}$ in various gauges.
\end{abstract}

$\ddagger$ Talk presented at 5th International Conference on the Renormalization Group, Helsinki, Finland, 30th August - 3rd September, 2005 


\section{Introduction}

The quantum field theory underlying the strong force of nature is widely accepted as being quantum chromodynamics (QCD) which is a non-abelian generalization of quantum electrodynamics. At large energy the constituent fields, the quarks and gluons, behave as free particles which is a property known as asymptotic freedom. In this case one performs calculations in QCD based on a vacuum which is empty and which is known as the perturbative vacuum. However, it is accepted [1, 2, 3, 4] that the true vacuum of QCD is more complicated and is not the perturbative one. An indication of this is that in the true vacuum gauge invariant operators condense. Indeed the two operators which receive wide attention are the operators $\alpha_{S} G_{\mu \nu}^{a} G^{a \mu \nu}$ and $\bar{\psi} \psi$, where $G_{\mu \nu}^{a}$ is the gluon field strength, $\psi$ is the quark field and $\alpha_{S}$ is the stong coupling constant. Consequently, it is possible to incorporate the vacuum expectation values $\left\langle\alpha_{S} G_{\mu \nu}^{a} G^{a \mu \nu}\right\rangle$ and $\langle\bar{\psi} \psi\rangle$ into the operator product expansion in order to determine the effects they have in the measurements of physical quantities and QCD sum rules, [5]. Indeed it is possible to extract numerical estimates for them. Whilst these operators are the main ones of interest, it has been pointed out more recently that additionally one can construct a dimension two operator in QCD which is gauge invariant, 6, 7, 8]. However, this is also believed to condense giving rise to $O\left(1 / Q^{2}\right)$ power corrections in the operator product expansion and other quantities, 9, 10, 11. Specifically the operator is

$$
\tilde{A}_{\mu}^{2}=\left[\min _{\{U\}} \int d^{4} x\left(A_{\mu}^{U}\right)^{2}\right] \mathcal{V}^{-1}
$$

where $U$ represents the set of all gauge configurations and $\tilde{A}_{\mu}^{a}$ is constructed in such a way that it is in fact gauge invariant. Consequently, unlike $G_{\mu \nu}^{a} G^{a \mu \nu}$ and $\bar{\psi} \psi$, the operator is non-local but can be written in terms of the usual gluon gauge field yielding a power series in $g$ when evaluated explicitly. This dimension two operator has been the subject of intense study in recent years, mostly from the point of view of trying to estimate a value for its vacuum expectation value, [12, 13, 14, 15, 16, 17, 18, 19, 20, 21, 22, 23, 24, 25, 26]. Further, the role a nonvanishing vacuum expectation value of a dimension two operator had on the estimate of glueball masses in the Coulomb gauge had been discussed earlier in [27].

Having a non-zero vacuum expectation value for this operator has interesting implications for trying to understand the properties of QCD and for phenomenology. One area of study has been on the lattice where there appears to be numerical evidence for $1 / Q^{2}$ power corrections in a variety of quantities [9, 10, 11]. For example, an effective strong coupling constant, $\alpha_{S}^{\mathrm{eff}}\left(Q^{2}\right)$, requires a $1 / Q^{2}$ correction to correctly fit lattice data in the range $2-6 \mathrm{GeV},[28,29]$. This necessitates a dimension two object on dimensional grounds. Another consequence is that such a dimension two condensate would imply that the gluon has an effective mass which is generated dynamically, 13, 14. Estimates for the value of such a mass have been summarized in table 15 of Field's article [30]. These have been extracted from phenomenology where one includes a gluon with a mass in order to fit experimental data more accurately. 
However, one of the main interests in understanding gluon mass is its relationship to the confinement mechanism. Whilst there are various ideas about what underlies this property of QCD and the strong force, the actual situation has not been determined yet. One point of view is that of abelian dominance [31, 32, 33. Essentially this is based on the premise that in the infrared the abelian sector of the gluon field dominates. It is then believed that the infrared sector of QCD could be described by a dual superconductor, whereby a monopole condensation would give rise to confinement via the dual Meissner effect. Moreover, in the context of the generation of an effective gluon mass, one viewpoint is that in the infrared the gluons associated with the centre of the colour group remain massless whilst the off-diagonal gluons gain a mass dynamically. Indeed there appears to be some preliminary lattice evidence for such a scenario, [34, 35]. To investigate such a hypothesis in QCD from a field theory point of view requires both a calculational technique to handle dimension two operator condensation as well as a way of focusing on the centre gluon fields. For the former, the local composite operator (LCO) method has been developed both for QCD, 8], and for models such as the two dimensional Gross-Neveu model [36, 37, where one has the exact mass gap to justify the approach. To examine the differing nature of the gluon field, one can choose to fix in the maximal abelian gauge (MAG) where the gauge fixing differentiates between centre and off-diagonal gluons. In the main in this article we review the procedures and recent results in using the LCO method to study the consequences of the condensation of a dimension two operator in QCD in various gauges, concentrating on those aspects which relate to the renormalization group which underpins the technique.

\section{Background}

Before detailing the LCO approach it is worth recalling the background to the problem of gluon mass in Yang-Mills theories. One early study was that of Curci and Ferrari in 38. where they constructed a Lagrangian with a gluon and ghost mass with a nonlinear gauge fixing. In particular the Lagrangian is

$$
\begin{aligned}
L= & -\frac{1}{4} G_{\mu \nu}^{A} G^{A \mu \nu}-\frac{1}{2 \alpha}\left(\partial^{\mu} A_{\mu}^{A}\right)^{2}+\frac{m^{2}}{2} A_{\mu}^{A} A^{A \mu} \\
& +\partial_{\mu} \bar{c}^{A} \partial^{\mu} c^{A}-\alpha m^{2} \bar{c}^{A} c^{A}-\frac{g}{2} f^{A B C} A_{\mu}^{A} \bar{c}^{B} \overleftrightarrow{\partial^{\mu}} c^{C} \\
& +\frac{\alpha g^{2}}{8} f^{E A B} f^{E C D} \bar{c}^{A} c^{B} \bar{c}^{C} c^{D}+i \bar{\psi} \not D \psi-m_{q} \bar{\psi} \psi
\end{aligned}
$$

where $A_{\mu}^{A}, c^{A}$ and $\psi^{i I}$ are the respective gluon, ghost and quark fields, $1 \leq A \leq N_{A}$, $1 \leq I \leq N_{F}$ and $1 \leq i \leq N_{f}$ with $N_{A}$ and $N_{F}$ the respective dimensions of the adjoint and fundamental representations, $N_{f}$ is the number of quarks, $T^{A}$ are the generators of the colour group whose structure constants are $f^{A B C}$ and the field strength is given by $G_{\mu \nu}^{A}=\partial_{\mu} A_{\nu}^{A}-\partial_{\nu} A_{\nu}^{A}-g f^{A B C} A_{\mu}^{B} A_{\nu}^{C}$ where $g$ is the coupling constant. In the case when the gluon mass $m$ is zero, the Lagrangian is regarded as QCD fixed in the Curci-Ferrari gauge. It gives rise to a different gluon-ghost interaction from that of the usual linear 
covariant gauge fixing. In addition there is a quartic ghost interaction which does not invalidate the renormalizability of the Lagrangian. When $m$ is non-zero one has a mass for both the gluon and the ghost where the respective gluon and ghost propagators are

$$
-\frac{\delta^{A B}}{\left(k^{2}+m^{2}\right)}\left[\eta^{\mu \nu}-\frac{(1-\alpha) k^{\mu} k^{\nu}}{\left(k^{2}+\alpha m^{2}\right)}\right] \text { and } \frac{\delta^{A B}}{\left(k^{2}+\alpha m^{2}\right)} .
$$

Whilst the Lagrangian is no longer invariant under gauge transformations, it is in fact (on-shell) BRST invariant for non-zero $m$, [38]. This latter property suggests it is a reasonable candidate for studying models with gluon mass. However, the initial interest in this model had to be tempered with the realization that whilst one has BRST invariance the BRST charge is not nilpotent since its square is propotional to $m^{2}$. Consequently one does not have a unitary theory and negative norm states can be constructed to demonstrate this [39, 40]. Aside from these limitations the CurciFerrari model has several important properties. One is that the presence of a mass for the gluon provides a natural infrared regulator in the theory. Indeed it has been renormalized explicitly at two loops, [41, 42. Therefore, it could be a useful tool in extracting renormalization constants where there are potential infrared problems. More importantly though the resurgence of interest in this model rests in its relationship to other gauges. In the case where $\alpha=0$, the Curci-Ferrari gauge reduces to the usual Landau gauge, 38. However, if one examines the off-diagonal sector of QCD fixed in the maximal abelian gauge (MAG), it transpires that that sector is precisely QCD fixed in the Curci-Ferrari gauge, [13]. Therefore, the Curci-Ferrari model can be used as a laboratory for investigating the problem of abelian dominance in QCD and the dynamical generation of mass for the off-diagonal gluons. Whilst the main disadvantage of the Curci-Ferrari model is the presence of a classical gluon mass leading to loss of unitarity, if a mass was generated dynamically by the condensation of a dimension two (BRST or gauge invariant) operator, then the unitarity issue may be circumvented.

\section{LCO method}

The LCO method is a procedure for including low dimension operators, such as $\frac{1}{2} A_{\mu}^{A} A^{A \mu}$, in the underlying quantum field theory and determining its effective potential. In this way one can examine to what extent the operator condenses by calculating whether the energy of the true vacuum when the operator is present is less than that of the true vacuum in its absence. For QCD it turns out that it leads to a modification of the Lagrangian so that new interactions are introduced which lead to an effective gluon mass. Part of the justification in applying the LCO method to QCD in a variety of gauges, such as the Landau, Curci-Ferrari or MAG, lies in the treatment of the two dimensional $O(N)$ Gross-Neveu model. There the mass gap is known exactly and the LCO approach obtains values for the mass gap to a few percent for a large range of $N$, 36, 37.

We now summarise the application of the method in the case of QCD in the Landau gauge. One of the advantages of this gauge is that the gauge invariant non-local operator 
(11) truncates to a single term local composite operator $\frac{1}{2} A_{\mu}^{A} A^{A \mu}$, 8 . In this instance one couples the operator to a source $J$ yielding the energy functional $W[J]$

$$
e^{-W[J]}=\int \mathcal{D}\left[A_{\mu} \psi \bar{\psi} c \bar{c}\right] \exp \left[\int d^{d} x\left(L_{\mathrm{gf}}-\frac{1}{2} Z_{m} J A_{\mu}^{A 2}+\frac{1}{2}(\xi+\delta \xi) J^{2}\right)\right] .
$$

From this, $W[J]$ satisfies a renormalization group equation

$$
\left[\mu \frac{\partial}{\partial \mu}+\beta(g) \frac{\partial}{\partial g^{2}}-\gamma_{m}(g) \int J \frac{\partial}{\partial J}+\eta(g, \xi) \frac{\partial}{\partial \xi}\right] W[J]=0
$$

where $\gamma_{m}(g)$ is the anomalous dimension of the operator derived from the corresponding renormalization constant $Z_{m}$ and $\mu$ is the renormalization scale introduced when one uses dimensional regularization in $d=4-2 \epsilon$ dimensions which is the regularization employed here. To ensure renormalizability one requires the additional term quadratic in $J$. This is because the vacuum energy in the presence of the operator is divergent with divergences proportional to $J^{2}$ appearing. The coefficient of $J^{2}$ is defined as $\xi$ where $\delta \xi$ is the counterterm and is at present not fixed, 8]. However, one can define a renormalization group function for the infinities associated with the $J^{2}$ term which are encoded in the related quantities $\eta(g, \xi)$ and $\delta(g)$ by

$$
\begin{aligned}
& \eta(g, \xi)=\mu \frac{\partial \xi}{\partial \mu} \mid=2 \gamma_{m}(g) \xi+\delta(g) \\
& \delta(g)=\left(2 \epsilon+2 \gamma_{m}(g)-\beta(g) \frac{\partial}{\partial g^{2}}\right) \delta \xi .
\end{aligned}
$$

In order to have a homogeneous renormalization group equation for $W[J]$ the as yet undetermined parameter $\xi$ must satisfy

$$
\beta(g) \frac{d \xi}{d g^{2}}=2 \gamma_{m}(g) \xi+\delta(g)
$$

whence

$$
\left[\mu \frac{\partial}{\partial \mu}+\beta(g) \frac{\partial}{\partial g^{2}}-\gamma_{m}(g) \int J \frac{\partial}{\partial J}\right] W[J]=0 .
$$

Therefore solving (7) will determine $\xi(g)$ once $\gamma_{m}(g)$ and $\delta(g)$ are known and this ensures that $\xi(g)$ runs as $g(\mu)$ runs. More importantly the homogeneity of (8) ensures that one retains an energy interpretation so that an effective action and thence an effective potential can be constructed for the operator in question, [8, 36, 37].

For practical calculations it would be more appropriate to have a functional with a linear source. This can be achieved by a Hubbard-Stratonovich transformation which introduces a scalar field $\sigma$ via

$$
1=\int \mathcal{D} \sigma \exp \left(-\int\left[a_{1} \sigma+a_{2} A_{\mu}^{A 2}+a_{3} J\right]^{2}\right)
$$

where the coefficients $a_{i}$ are chosen appropriately to cancel the $J^{2}$ term. Consequently in the Landau gauge one obtains the renormalizable Lagrangian for $\sigma$, and therefore the operator $\frac{1}{2} A_{\mu}^{A} A^{A \mu}$ as

$$
L^{\sigma}=L_{\mathrm{gf}}-\frac{\sigma^{2}}{2 g^{2} \xi(g) Z_{\xi}}+\frac{Z_{m}}{2 g \xi(g) Z_{\xi}} \sigma A_{\mu}^{A} A^{A \mu}-\frac{Z_{m}^{2}}{8 \xi(g) Z_{\xi}}\left(A_{\mu}^{A} A^{A \mu}\right)^{2} .
$$


Once the expressions for $\gamma_{m}(g)$ and $\xi(g)$ are known then the effective potential can be constructed. Though for a two loop potential one requires the renormalization group functions at three loops.

\section{Three loop renormalization}

As the LCO method relies on requiring explicit values of the renormalization group functions at large loop order it is important to study the renormalization of QCD in the context of the operator $\frac{1}{2} A_{\mu}^{A} A^{A \mu}$ and in various gauges. For the Landau gauges all the information to construct $\gamma_{m}(g)$ in fact is in place. This is due to an observation from explicit calculations and the general formalism of algebraic renormalization which demonstrate that to all orders in perturbation theory the anomalous dimension of $\frac{1}{2} A_{\mu}^{A} A^{A \mu}$ is not independent, [43. More specifically

$$
\gamma_{m}(g)=\gamma_{A}(g)+\gamma_{c}(g)
$$

in the Landau gauge. This curious property is not restricted to this gauge as in the MAG the anomalous dimension of the analogous dimension two operator, based on offdiagonal fields, involves the anomalous dimensions of the diagonal gluon and diagonal ghost, [26]. In the more general Curci-Ferrari gauge we have observed a generalization of (11) in an explicit three loop $\overline{\mathrm{MS}}$ renormalization, [44, which is

$$
\gamma_{m}(g)=\gamma_{A}(g)+\gamma_{c}(g)-2 \gamma_{\alpha}(g)
$$

where we note that unlike the linear covariant gauges the anomalous dimension corresponding to the renormalization of the gauge parameter, $\gamma_{\alpha}(g)$, is non-zero. Unfortunately it has not been established whether this latter relation remains valid beyond three loops.

One issue which arises when one is working with the renormalization of operators and this is the question of operator mixing. The BRST invariant mass operator involves the two terms $\frac{1}{2} A_{\mu}^{A} A^{A \mu}$ and $\bar{c}^{A} c^{A}$. In principle it could be the case that the combination $\mathcal{O}=\frac{1}{2} A_{\mu}^{A} A^{A \mu}-\alpha \bar{c}^{A} c^{A}$ does not renormalize multiplicatively. However, in linear covariant gauges it turns out that the mixing matrix is triangular, 45, but not in the Curci-Ferrari gauge. Indeed in [46 the one loop mixing matrix for $\mathcal{O}_{i}$ was determined where $\mathcal{O}_{1}=\frac{1}{2} A_{\mu}^{A} A^{A \mu}$ and $\mathcal{O}_{2}=\bar{c}^{A} c^{A}$. We have extended that calculation to two loops for potential future extensions of the operator product expansion analysis of [46]. If we set

$$
\mathcal{O}_{o i}=Z_{i j} \mathcal{O}_{j}
$$

where $Z_{i j}$ is the mixing matrix of renormalization constants. With

$$
\gamma_{i j}(g)=\mu \frac{\partial}{\partial \mu} \ln Z_{i j}
$$

then we have

$$
\gamma_{11}=\left(\frac{35}{12}+\frac{\alpha}{4}\right) C_{A} a+\left(\frac{449}{48}+\frac{11 \alpha}{16}+\frac{3 \alpha^{2}}{16}\right) C_{A}^{2} a^{2}+O\left(a^{3}\right)
$$




$$
\begin{aligned}
& \gamma_{12}=-\frac{\alpha^{2}}{4} C_{A} a-\left(\frac{5 \alpha^{2}}{16}+\frac{\alpha^{3}}{8}\right) C_{A}^{2} a^{2}+O\left(a^{3}\right) \\
& \gamma_{21}=\frac{\alpha}{2} C_{A} a-\left(\frac{11}{8}+\frac{\alpha}{4}\right) C_{A}^{2} a^{2}+O\left(a^{3}\right) \\
& \gamma_{22}=\left(\frac{3}{4}-\frac{\alpha}{4}\right) C_{A} a+\left(\frac{95}{48}+\frac{\alpha}{16}-\frac{\alpha^{2}}{8}\right) C_{A}^{2} a^{2}+O\left(a^{3}\right)
\end{aligned}
$$

where $a=g^{2} /\left(16 \pi^{2}\right), T^{A} T^{A}=C_{F} I, f^{A C D} f^{B C D}=C_{A} \delta^{A B}$ and $\operatorname{Tr}\left(T^{A} T^{B}\right)=T_{F} \delta^{A B}$. These results were obtained by renormalizing the operators in the Curci-Ferrari model where there is a non-zero infrared regulating mass, by inserting them into gluon and ghost two-point functions. The Curci-Ferrari model has the advantage that external momenta can be nullified without introducing spurious infrared infinities as a consequence. It remains merely to extract the infinities from the resultant vacuum bubbles. Not only did we reproduce the one loop matrix of Kondo, [46], but we obtained the result that

$$
\gamma_{m}(g)=\gamma_{11}(g)-\alpha \gamma_{21}(g)
$$

at two loops, thus verifying that $\mathcal{O}$ is multiplicatively renormalizable at this order.

For three loop calculations the massive propagator approach is tedious and we produced an equivalent method based on the MiNCER algorithm, 47, 48, which is implemented in the symbolic manipulation language Form, 49]. For example, one can determine $\delta \xi$ by treating the term $\frac{1}{2} J A_{\mu}^{A} A^{A \mu}$ of (10) as an interaction and computing the divergence structure of the $J$ two-point function with massless internal fields but not internal $J$ propagators, 24]. The explicit Feynman diagrams are generated automatically with the QGRAF package, [50]. The MINCER algorithm was especially appropriate for the three loop renormalization of QCD in the MAG, [51], which is necessary for the construction of the two loop effective potential for the analogous dimension two BRST invariant operator. Unlike the linear covariant gauges the full three loop renormalization was determined only recently, 51]. Moreover, it was a significantly large computation requiring the evaluation of 37322 Feynman diagrams compared with of the order of 1000 for a linear covariant gauge three loop renormalization.

Briefly, the MAG involves the decomposition of the gauge field $A_{\mu}^{A}$ into diagonal and off-diagonal sectors

$$
A_{\mu}^{A} T^{A}=A_{\mu}^{a} T^{a}+A_{\mu}^{i} T^{i}
$$

where $1 \leq a \leq N_{A}^{o}$ and $1 \leq i \leq N_{A}^{d}$ and $N_{A}^{d}$ is the dimension of the centre of the colour group and $N_{A}^{o}$ is the dimension of the remainder with $N_{A}^{d}+N_{A}^{o}=N_{A}$. Notationally we will reserve $i, j, k$ and $l$ for indices on objects which lie in the centre of the group and the remaining lower case Roman letters for off-diagonal objects. Consequently, the MAG gauge fixing term is, [26],

$$
L_{\mathrm{gf}}^{\mathrm{MAG}}=\delta \bar{\delta}\left[\frac{1}{2} A_{\mu}^{a} A^{a \mu}+\frac{1}{2} \alpha \bar{c}^{a} c^{a}\right]+\delta\left[\bar{c}^{i} \partial^{\mu} A_{\mu}^{i}\right]
$$

where $\delta$ and $\bar{\delta}$ are the BRST and anti-BRST transformations. The remaining gauge freedom associated with the diagonal gluons is fixed by using a Landau gauge. Further, 
the analogous mass operator to $\mathcal{O}$ is

$$
\mathcal{O}^{\mathrm{MAG}}=\frac{1}{2} A_{\mu}^{a} A^{a \mu}-\alpha \bar{c}^{a} c^{a}
$$

To renormalize the resultant Lagrangian

$$
\begin{aligned}
L_{\mathrm{gf}}^{\mathrm{MAG}}=-\frac{1}{2 \alpha} & \left(\partial^{\mu} A_{\mu}^{a}\right)^{2}-\frac{1}{2 \bar{\alpha}}\left(\partial^{\mu} A_{\mu}^{i}\right)^{2}+\bar{c}^{a} \partial^{\mu} \partial_{\mu} c^{a}+\bar{c}^{i} \partial^{\mu} \partial_{\mu} c^{i} \\
+g[ & f^{a b k} A_{\mu}^{a} \bar{c}^{k} \partial^{\mu} c^{b}-f^{a b c} A_{\mu}^{a} \bar{c}^{b} \partial^{\mu} c^{c} \\
& -\frac{1}{\alpha} f^{a b k} \partial^{\mu} A_{\mu}^{a} A_{\nu}^{b} A^{k \nu}-f^{a b k} \partial^{\mu} A_{\mu}^{a} c^{b} \bar{c}^{k} \\
& \left.-\frac{1}{2} f^{a b c} \partial^{\mu} A_{\mu}^{a} \bar{c}^{b} c^{c}-2 f^{a b k} A_{\mu}^{k} \bar{c}^{a} \partial^{\mu} \bar{c}^{b}-f^{a b k} \partial^{\mu} A_{\mu}^{k} \bar{c}^{b} c^{c}\right] \\
+g^{2}[ & f_{d}^{a c b d} A_{\mu}^{a} A^{b \mu} \bar{c}^{c} c^{d}-\frac{1}{2 \alpha} f_{o}^{a k b l} A_{\mu}^{a} A^{b \mu} A_{\nu}^{k} A^{l \nu} \\
& +f_{o}^{a d c j} A_{\mu}^{a} A^{j \mu} \bar{c}^{c} c^{d}-\frac{1}{2} f_{o}^{a j c d} A_{\mu}^{a} A^{j \mu} \bar{c}^{c} c^{d} \\
& +f_{o}^{a j c l} A_{\mu}^{a} A^{j \mu} \bar{c}^{c} c^{l}+f_{o}^{a l c j} A_{\mu}^{a} A^{j \mu} \bar{c}^{c} c^{l}-f_{o}^{c j d i} A_{\mu}^{i} A^{j \mu} \bar{c}^{c} c^{d} \\
& -\frac{\alpha}{4} f_{d}^{a b c d} \bar{c}^{a} \bar{c}^{b} c^{c} c^{d}-\frac{\alpha}{8} f_{o}^{a b c d} \bar{c}^{a} \bar{c}^{b} c^{c} c^{d} \\
& +\frac{\alpha}{8} f_{o}^{a c b d} \bar{c}^{a} \bar{c}^{b} c^{c} c^{d}-\frac{\alpha}{4} f_{o}^{a b c l} \bar{c}^{a} \bar{c}^{b} c^{c} c^{l} \\
& \left.+\frac{\alpha}{4} f_{o}^{a c b l} \bar{c}^{a} \bar{c}^{b} c^{c} c^{l}-\frac{\alpha}{4} f_{o}^{a l b c} \bar{c}^{a} \bar{c}^{b} c^{c} c^{l}+\frac{\alpha}{2} f_{o}^{a k b l} \bar{c}^{a} \bar{c}^{b} c^{k} c^{l}\right]
\end{aligned}
$$

where

$$
f_{d}^{A B C D}=f^{i A B} f^{i C D}, f_{o}^{A B C D}=f^{e A B} f^{e C D}
$$

one introduces renormalization constants via, [26, 52, 53, 54, 155, 56],

$$
\begin{aligned}
& A_{\mathrm{O}}^{a \mu}=\sqrt{Z_{A}} A^{a \mu}, A_{\mathrm{O}}^{i \mu}=\sqrt{Z_{A^{i}}} A^{i \mu} \\
& c_{\mathrm{O}}^{a}=\sqrt{Z_{c}} c^{a}, \quad \bar{c}_{\mathrm{O}}^{a}=\sqrt{Z_{c}} \bar{c}^{a} \\
& c_{\mathrm{O}}^{i}=\sqrt{Z_{c^{i}}} c^{i}, \quad \bar{c}_{\mathrm{O}}^{i}=\frac{\bar{c}^{i}}{\sqrt{Z_{c^{i}}}}, \quad \psi_{\mathrm{O}}=\sqrt{Z_{\psi}} \psi, \\
& g_{\mathrm{O}}=\mu^{\epsilon} Z_{g} g, \quad \alpha_{\mathrm{O}}=Z_{\alpha}^{-1} Z_{A} \alpha, \bar{\alpha}_{\mathrm{O}}=Z_{\alpha^{i}}^{-1} Z_{A^{i}} \bar{\alpha} .
\end{aligned}
$$

However, it is crucial to note that this choice is determined by the application of the algebraic renormalization method, [26]. This shows, for example, that the diagonal ghost two-point function is finite to all orders and implies that its anomalous dimension must be deduced from another Green's function such as the $A_{\mu}^{a} \bar{c}^{i} c^{b}$ vertex. Also, the diagonal gluon anomalous dimension is not independent since its associated renormalization constant is equivalent to that for the coupling constant, [26]. A similar feature occurs in the background field gauge, [57, 58, 59, 60]. Whilst the application of the MiNCER algorithm is straightforward to extract all the necessary renormalization constants, the bulk of the work lies in symbolically implementing the underlying group theory relations founded upon the elementary equations

$$
f^{i j k}=f^{a j k}=0, f^{a b k} \neq 0, f^{a b c} \neq 0 .
$$


Consequently one obtains representative anomalous dimensions of the following form in the $\overline{\mathrm{MS}}$ scheme

$$
\begin{aligned}
& \gamma_{c^{i}}(a)=\frac{1}{4 N_{A}^{o}}\left[N_{A}^{o}\left((-\alpha-3) C_{A}\right)+N_{A}^{d}\left((-2 \alpha-6) C_{A}\right)\right] a \\
&+\frac{1}{96 N_{A}^{o 2}}\left[N_{A}^{o 2}\left(\left(-6 \alpha^{2}-66 \alpha-190\right) C_{A}^{2}+80 C_{A} T_{F} N_{f}\right)\right. \\
&+N_{A}^{o} N_{A}^{d}\left(\left(-54 \alpha^{2}-354 \alpha-323\right) C_{A}^{2}+160 C_{A} T_{F} N_{f}\right) \\
&\left.+N_{A}^{d^{2}}\left(\left(-60 \alpha^{2}-372 \alpha+510\right) C_{A}^{2}\right)\right] a^{2} \\
&+\frac{1}{6912 N_{A}^{o 3}}\left[N _ { A } ^ { o 3 } \left(\left(-162 \alpha^{3}-2727 \alpha^{2}-2592 \zeta_{3} \alpha-18036 \alpha\right.\right.\right. \\
&\left.-1944 \zeta_{3}-63268\right) C_{A}^{3}+\left(6912 \alpha+62208 \zeta_{3}+6208\right) C_{A}^{2} T_{F} N_{f} \\
&\left.+\left(-82944 \zeta_{3}+77760\right) C_{A} C_{F} T_{F} N_{f}+8960 C_{A} T_{F}^{2} N_{f}^{2}\right) \\
&+ N_{A}^{o 2} N_{A}^{d}\left(\left(-2754 \alpha^{3}+648 \zeta_{3} \alpha^{2}-28917 \alpha^{2}-4212 \zeta_{3} \alpha\right.\right. \\
&\left.-69309 \alpha+37260 \zeta_{3}-64544\right) C_{A}^{3} \\
&+\left(25488 \alpha+103680 \zeta_{3}-13072\right) C_{A}^{2} T_{F} N_{f} \\
&\left.+\left(-165888 \zeta_{3}+155520\right) C_{A} C_{F} T_{F} N_{f}+17920 C_{A} T_{F}^{2} N_{f}^{2}\right) \\
&+N_{A}^{o} N_{A}^{d^{2}}\left(\left(-7884 \alpha^{3}+22680 \zeta_{3} \alpha^{2}-84564 \alpha^{2}+97524 \zeta_{3} \alpha\right.\right. \\
&\left.-47142 \alpha+433836 \zeta_{3}-56430\right) C_{A}^{3} \\
&\left.+\left(25056 \alpha-124416 \zeta_{3}-18144\right) C_{A}^{2} T_{F} N_{f}\right) \\
&+N_{A}^{d^{3}}\left(\left(-6480 \alpha^{3}+34992 \zeta_{3} \alpha^{2}-70092 \alpha^{2}+8424 \zeta_{3} \alpha\right.\right. \\
&\left.\left.\left.+114912 \alpha+77112 \zeta_{3}-161028\right) C_{A}^{3}\right)\right] a^{3}+O\left(a^{4}\right) \\
&
\end{aligned}
$$

and

$$
\begin{aligned}
\gamma_{\mathcal{O}}(a)= & \frac{1}{12 N_{A}^{o}}\left[N_{A}^{o}\left((-3 \alpha+35) C_{A}-16 T_{f} N_{f}\right)+N_{A}^{d}\left((-6 \alpha-18) C_{A}\right)\right] a \\
+ & \frac{1}{96 N_{A}^{o 2}}\left[N _ { A } ^ { o 2 } \left(\left(-6 \alpha^{2}-66 \alpha+898\right) C_{A}^{2}-560 C_{A} T_{f} N_{f}\right.\right. \\
& \left.-384 C_{F} T_{f} N_{f}\right) \\
+ & N_{A}^{o} N_{A}^{d}\left(\left(-54 \alpha^{2}-354 \alpha-323\right) C_{A}^{2}+160 C_{A} T_{f} N_{f}\right) \\
& \left.+N_{A}^{d^{2}}\left(\left(-60 \alpha^{2}-372 \alpha+510\right) C_{A}^{2}\right)\right] a^{2} \\
+ & \frac{1}{6912 N_{A}^{o 3}}\left[N _ { A } ^ { o 3 } \left(\left(-162 \alpha^{3}-2727 \alpha^{2}-2592 \zeta_{3} \alpha-18036 \alpha\right.\right.\right. \\
& \left.-1944 \zeta_{3}+302428\right) C_{A}^{3} \\
& +\left(6912 \alpha+62208 \zeta_{3}-356032\right) C_{A}^{2} T_{F} N_{f} \\
& +\left(-82944 \zeta_{3}-79680\right) C_{A} C_{F} T_{F} N_{f}+49408 C_{A} T_{F}^{2} N_{f}^{2} \\
& \left.+13824 C_{F}^{2} T_{F} N_{f}+33792 C_{F} T_{F}^{2} N_{f}^{2}\right) \\
& +N_{A}^{o 2} N_{A}^{d}\left(\left(-2754 \alpha^{3}+648 \alpha^{2} \zeta_{3}-28917 \alpha^{2}\right.\right. \\
& \left.-4212 \alpha \zeta_{3}-69309 \alpha+37260 \zeta_{3}-64544\right) C_{A}^{3}
\end{aligned}
$$




$$
\begin{aligned}
& +\left(25488 \alpha+103680 \zeta_{3}-13072\right) C_{A}^{2} T_{F} N_{f} \\
& \left.+\left(-165888 \zeta_{3}+155520\right) C_{A} C_{F} T_{F} N_{f}+17920 C_{A} T_{F}^{2} N_{f}^{2}\right) \\
& +N_{A}^{o} N_{A}^{d^{2}}\left(\left(-7884 \alpha^{3}+22680 \alpha^{2} \zeta_{3}-84564 \alpha^{2}+97524 \alpha \zeta_{3}\right.\right. \\
& \left.-47142 \alpha+433836 \zeta_{3}-56430\right) C_{A}^{3}+\left(25056 \alpha-124416 \zeta_{3}\right. \\
& \left.-18144) C_{A}^{2} T_{F} N_{f}\right)+N_{A}^{d^{3}}\left(\left(-6480 \alpha^{3}+34992 \alpha^{2} \zeta_{3}-70092 \alpha^{2}\right.\right. \\
& \left.\left.\left.+8424 \alpha \zeta_{3}+114912 \alpha+77112 \zeta_{3}-161028\right) C_{A}^{3}\right)\right] a^{3}+O\left(a^{4}\right)
\end{aligned}
$$

for the MAG mass operator where $\zeta_{n}$ is the Riemann zeta function, [51]. In addition the three loop $\beta$-function correctly emerges from the diagonal gluon two-point function which is a strong check on the programming and computation since not only must it be independent of the gauge parameter $\alpha$ but also of the sector dimensions $N_{A}^{d}$ and $N_{A}^{o}$. Another useful check on this and the anomalous dimensions was the fact that the known Curci-Ferrari gauge anomalous dimensions, [11, 42, 44, emerge in the limit $N_{A}^{d} / N_{A}^{o} \rightarrow 0$. This is consistent with the relation of the Curci-Ferrari gauge to the MAG, [13].

\section{Results}

Having detailed the renormalization group aspects underlying the LCO formalism we now briefly summarize recent results of determining estimates for the gluon mass in various gauges, [8, 24, 26]. First, for the Landau gauge the effective potential for $\sigma$ is, [8, 24],

$$
\begin{aligned}
V(\sigma)=\frac{9 N_{A}}{2} \lambda_{1} \sigma^{\prime 2} & \\
+ & {\left[\frac{3}{64} \ln \left(\frac{g \sigma^{\prime}}{\bar{\mu}^{2}}\right)-C_{A}\left(\frac{351}{8} C_{F} \lambda_{1} \lambda_{2}-\frac{351}{16} C_{F} \lambda_{1} \lambda_{3}\right.\right.} \\
& \left.+\frac{249}{128} \lambda_{2}-\frac{27}{64} \lambda_{3}\right)+C_{A}^{2}\left(-\frac{81}{16} \lambda_{1} \lambda_{2}+\frac{81}{32} \lambda_{1} \lambda_{3}\right) \\
& \left.+\left(-\frac{13}{128}-\frac{207}{32} C_{F} \lambda_{2}+\frac{117}{32} C_{F} \lambda_{3}\right)\right] \frac{g^{2} N_{A} \sigma^{2}}{\pi^{2}}+O\left(g^{4}\right)
\end{aligned}
$$

where space has restricted us to the one loop expression and $\lambda_{1}=\left[13 C_{A}-8 T_{F} N_{f}\right]^{-1}$, $\lambda_{2}=\left[35 C_{A}-16 T_{F} N_{f}\right]^{-1}, \lambda_{3}=\left[19 C_{A}-8 T_{F} N_{f}\right]^{-1}$ and $\sigma=\frac{9 N_{A}}{\left(13 C_{A}-8 T_{F} N_{f}\right)} \sigma^{\prime}$. Examining the solution to $V^{\prime}(\sigma)=0$ there are two possibilities which are $\langle\sigma\rangle=0$ or $\langle\sigma\rangle \neq 0$. For the former this is the original classical vacuum but the latter corresponds to a new vacuum which has an energy lower than the former. Thus in the presence of the $\frac{1}{2} A_{\mu}^{A} A^{A \mu}$ operator the effective potential produces a new vacuum which is stable unlike the now unstable (perturbative) classical vacuum. Moreover, boundedness of the potential requires that $\left[13 C_{A}-8 T_{F} N_{F}\right]$ needs to be positive, 24]. Interestingly this corresponds to the Landau gauge one loop gluon anomalous dimension which has been suggested as part of the necessary criterion underlying confinement when that problem is considered from a renormalization group perspective, 61, 62. Consequently if one defines $m_{\text {eff }}^{2}=\sigma /(g \xi(g))$ as an effective gluon mass then for $S U(3)$ Yang-Mills 
$m_{\mathrm{eff}}=2.13 \Lambda_{\overline{\mathrm{MS}}}$ from the two loop potential [8, 24]. This is within $2 \%$ of the one loop estimate indicating a degree of stability in the approach. As an alternative one can compute the gluon pole mass by first redefining $\sigma^{\prime}$ in terms of the pole mass and demanding the alternative condition, 63, 64],

$$
\frac{d V\left(m_{\text {pole }}\right)}{d m_{\text {pole }}}=0 .
$$

Interestingly at one loop this produces a Yang-Mills mass which is independent of the renormalization scale, [63. Though at two loops, like the effective mass of [8], the pole mass derived from the effective potential is scale dependent.

For the MAG the analysis is not fully complete as only the one loop potential for $S U(2)$ has been determined, [26]. However, the situation there is encouraging in that for pure Yang-Mills a mass is generated for the off-diagonal gluons which is $m_{\mathrm{eff}}=2.25 \Lambda_{\overline{\mathrm{MS}}}$. This is not dissimilar to the Landau gauge $S U(2)$ estimate of $m_{\mathrm{eff}}=2.03 \Lambda_{\overline{\mathrm{MS}}}$. In addition the off-diagonal ghost and diagonal gluon remain massless. The appearance of the potential diagonal gluon mass operator, $\frac{1}{2} A_{\mu}^{i} A^{i \mu}$, in the LCO action used for the MAG, [26], is excluded by the diagonal $U(1)$ Ward identity deriving from the algebraic renormalization analysis, [26]. We are unable to prove the renormalizability of the action supplemented with a mass term like $\frac{1}{2} \mathcal{J} A_{\mu}^{i} A^{i \mu}$. Indeed overall this mass generation scenario appears to be consistent with $S U(2)$ lattice studies in the maximal abelian gauge, [34, 35, 65].

\section{Conclusions}

We conclude with various observations. First, we have given an overview of the current status of the application of the local composite operator method to the condensation of a renormalizable dimension two operator in $\mathrm{QCD}$ in various gauges, concentrating on the underlying renormalization group aspects. One main feature is the construction of a two loop effective potential for the operator which requires knowledge of the three loop anomalous dimensions of QCD. Whilst these are known for linear covariant gauges, to examine the abelian dominance hypothesis in the infrared, the more appropriate maximal abelian gauge needs to be used. This has required the full three loop renormalization of $\mathrm{QCD}$ in the MAG, which is a significantly larger computation from the point of view of the number of Feynman diagrams to be evaluated. Moreover, it opens up the possibility of examining the generation of a mass for the off-diagonal gluon at the two loop level and for gauge groups other than $S U(2)$. Whilst this may seem to be a feature of this gauge, the issue of whether one can access abelian dominance in a covariant gauge, where the properties of the centre of the group are not explicit in the fields one uses, has recently been studied using the LCO formalism, [66]. In particular the presence of ghost condensates in $S U(2)$ appears to be central in the dynamical generation of a mass for the off diagonal gluons which is different from that of the diagonal gluons. Indeed there would appear to be evidence from a recent lattice study to support this point of view, 67. 


\section{Acknowledgments}

We gratefully acknowledge the financial support which was provided by the Conselho

Nacional de Desenvolvimento Científico e Tecnológico (CNPq-Brazil), the Faperj, Fundação de Amparo à Pesquisa do Estado do Rio de Janeiro, the SR2-UERJ, the Coordenação de Aperfeiçoamento de Pessoal de Nível Superior (CAPES), PPARC for a research studentship and the Special Research Fund of Ghent University.

\section{References}

[1] G.K. Savvidy, Phys. Lett. B71 (1977), 133.

[2] V.P. Gusynin \& V.A. Miransky, Phys. Lett. B76 (1978), 585.

[3] R. Fukuda \& T. Kugo, Prog. Theor. Phys. 60 (1978), 565.

[4] R. Fukuda, Phys. Lett. B73 (1978), 33; Phys. Lett. B74 (1978), 433.

[5] M.A. Shifman, A.I. Vainshtein \& V.I. Zakharov, Nucl. Phys. B147 (1979), 385.

[6] F.V. Gubarev, L. Stodolsky \& V.I. Zakharov, Phys. Rev. Lett. 86 (2001), 2220.

[7] F.V. Gubarev \& V.I. Zakharov, Phys. Lett. B501 (2001), 28.

[8] H. Verschelde, K. Knecht, K. van Acoleyen \& M. Vanderkelen, Phys. Lett. B516 (2001), 307.

[9] P. Boucaud, A. Le Yaouanc, J.P. Leroy, J. Micheli, O. Pène \& J. Rodriguez-Quintero, Phys. Rev. D63 (2001), 114003.

[10] P. Boucaud, J.P. Leroy, A. Le Yaounac, J. Micheli, O. Pène, F. De Soto, A. Donini, H. Moutarde \& J. Rodriguez-Quintero, Phys. Rev. D66 (2002), 034504.

[11] P. Boucaud, J.P. Leroy, A. Le Yaounac, A.Y. Lokhov, J. Micheli, O. Pène, J. Rodriguez-Quintero \& C. Roiesnel, hep-lat/0507005.

[12] K.-I. Kondo \& T. Shinohara, Phys. Lett. B491 (2000), 263.

[13] K.-I. Kondo, Phys. Lett. B514 (2001), 335.

[14] K.-I. Kondo, hep-ph/0110013

[15] M. Schaden, hep-th/9909011.

[16] M. Schaden, hep-th/0108034

[17] U. Ellwanger \& N. Wschebor, Int. J. Mod. Phys. A18 (2003), 1595.

[18] D. Dudal \& H. Verschelde, J. Phys. A36 (2003), 8507.

[19] V.E.R. Lemes, M.S. Sarandy \& S.P. Sorella, J. Phys. A36 (2003), 7211.

[20] V.E.R. Lemes, M.S. Sarandy \& S.P. Sorella, Mod. Phys. Lett. A18 (2003), 711.

[21] V.E.R. Lemes, M.S. Sarandy \& S.P. Sorella, Ann. Phys. 308 (2003), 1.

[22] D. Dudal, H. Verschelde, V.E.R. Lemes, M.S. Sarandy, S.P. Sorella \& M. Picariello, Ann. Phys. 308 (2003), 62.

[23] D. Dudal, A.R. Fazio, V.E.R. Lemes, M. Picariello, M.S. Sarandy, S.P. Sorella \& H. Verschelde, Nucl. Phys. Proc. Suppl. 127C (2004), 154.

[24] R.E. Browne \& J.A. Gracey, JHEP 0311 (2003), 029.

[25] D. Dudal, H. Verschelde, J.A. Gracey, V.E.R. Lemes, M.S. Sarandy, R.F. Sobreiro \& S.P. Sorella, JHEP 0401 (2004), 044.

[26] D. Dudal, J.A. Gracey, V.E.R. Lemes, M.S. Sarandy, R.F. Sobreiro, S.P. Sorella \& H. Verschelde, Phys. Rev D70 (2004), 114038.

[27] J. Greensite \& M.B. Halpern, Nucl. Phys. B271 (1986), 379.

[28] P. Boucaud, G. Burgio, F. Di Renzo, J.P. Leroy, J. Micheli, C. Parrinello, O. Pène, C. Pittori, J. Rodriguez-Quintero, C. Roiesnel \& K. Sharkey, JHEP 0004 (2000), 006.

[29] D. Becirevic, P. Boucaud, F. De Soto, A. Le Yaouanc, J.P. Leroy, J. Micheli, O. Pène, J. RodriguezQuintero \& C. Roiesnel, Nucl. Phys. Proc. Suppl. 106 (2002), 867.

[30] J.H. Field, Phys. Rev. D66 (2002), 013013. 
[31] Y. Nambu, Phys. Rev. D10 (1974), 4262; G. 't Hooft, High Energy Physics EPS Int. Conference,

Palermo 1975, ed. A. Zichichi; S. Mandelstam, Phys. Rept. 23 (1976), 245.

[32] G. 't Hooft, Nucl. Phys. B190 (1981), 455.

[33] Z.F. Ezawa \& A. Iwazaki, Phys. Rev. D25 (1982), 2681.

[34] K. Amemiya \& H. Suganuma, Phys. Rev. D60 (1999), 114509.

[35] V.G. Bornyakov, M.N. Chernodub, F.V. Gubarev, S.M. Morozov \& M.I. Polikarpov, Phys. Lett. B559 (2003), 214.

[36] H. Verschelde, Phys. Lett. B351 (1995), 242.

[37] H. Verschelde, S. Schelstraete \& M. Vanderkelen, Z. Phys. C76 (1997), 161.

[38] G. Curci \& R. Ferrari, Nuovo Cim. A32 (1976), 151.

[39] I. Ojima, Z. Phys. C13 (1982), 173.

[40] J. de Boer, K. Skenderis, P. van Nieuwenhuizen \& A. Waldron, Phys. Lett. B367 (1996), 175.

[41] J.A. Gracey, Phys. Lett. B525 (2002), 89.

[42] R.E. Browne \& J.A. Gracey, Phys. Lett. B540 (2002), 68.

[43] D. Dudal, H. Verschelde \& S.P. Sorella, Phys. Lett. B555 (2003), 126.

[44] J.A. Gracey, Phys. Lett. B552 (2003), 101.

[45] D. Dudal, H. Verschelde, V.E.R. Lemes, M.S. Sarandy, R.F. Sobreiro, S.P. Sorella, M. Picariello, J.A. Gracey, Phys. Lett. B569 (2003), 57.

[46] K.I. Kondo, T. Murakami, T. Shinohara \& T. Imai, Phys. Rev. D65 (2002), 085034.

[47] S.G. Gorishny, S.A. Larin, L.R. Surguladze \& F.K. Tkachov, Comput. Phys. Commun. 55 (1989), 381.

[48] S.A. Larin, F.V. Tkachov \& J.A.M. Vermaseren, "The Form version of Mincer", NIKHEF-H-91-18.

[49] J.A.M. Vermaseren, math-ph/0010025.

[50] P. Nogueira, J. Comput. Phys. 105 (1993), 279.

[51] J.A. Gracey, JHEP 0504 (2005), 012.

[52] H. Min, T. Lee \& P.Y. Pac, Phys. Rev. D32 (1985), 440.

[53] A.R. Fazio, V.E.R. Lemes, M.S. Sarandy \& S.P. Sorella, Phys. Rev. D64 (2001), 085003.

[54] T. Shinohara, T. Imai \& K.I. Kondo, Int. J. Mod. Phys. A18 (2003), 5733.

[55] K.I. Kondo \& T. Shinohara, Prog. Theor. Phys. 105 (2001), 649.

[56] T. Shinohara, Mod. Phys. Lett. A18 (2003), 1398.

[57] B.S. DeWitt, Phys. Rev. 162 (1967), 1195; G. 't Hooft, Acta Universitatis Wratislaviensis 368 (1976), 345, Proceedings of the 1975 Winter School of Theoretical Physics held in Karpacz; B.S. DeWitt, in Proceedings of Quantum Gravity II, eds C. Isham, R. Penrose \& S. Sciama, (Oxford, 1980), 449.

[58] D.G. Boulware, Phys. Rev. D23 (1981), 389.

[59] L.F. Abbott, Nucl. Phys. B185 (1981), 189.

[60] D.M. Capper \& A. MacLean, Nucl. Phys. B203 (1982), 413.

[61] K. Nishijima, Int. J. Mod. Phys. A9 (1994), 3799.

[62] M. Chaichian \& K. Nishijima, Eur. Phys. J. C22 (2001), 463.

[63] R.E. Browne \& J.A. Gracey, Phys. Lett. B597 (2004), 368.

[64] J.A. Gracey, Eur. Phys. J. C39 (2005), 61.

[65] M.N. Chernodub, K. Ishiguro, Y. Mori, Y. Nakamura, M.I. Polikarpov, T. Sekido, T. Suzuki \& V.I. Zakharov, Phys. Rev. D72 (2005), 074505.

[66] M.A.L. Capri, D. Dudal, J.A. Gracey, V.E.R. Lemes, R.F. Sobreiro, S.P. Sorella \& H. Verschelde, hep-th/0508216.

[67] A. Cucchieri, T. Mendes \& A. Mihari, hep-lat/0508028 